\title{
The gut microbiome: a key player in the complexity of amyotrophic lateral sclerosis (ALS)
}

Sarah L. Boddy ${ }^{1 \dagger}$, Ilaria Giovannelli ${ }^{1 \dagger}$, Matilde Sassani ${ }^{1 \dagger}$, Johnathan Cooper-Knock ${ }^{1}$, Michael P. Snyder ${ }^{2}$, Eran Segal ${ }^{3}$, Eran Elinav ${ }^{4,5}$, Lynne A. Barker ${ }^{6}$, Pamela J. Shaw ${ }^{1}$ and Christopher J. McDermott ${ }^{\text {* }^{*}}$

\begin{abstract}
Background: Much progress has been made in mapping genetic abnormalities linked to amyotrophic lateral sclerosis (ALS), but the majority of cases still present with no known underlying cause. Furthermore, even in families with a shared genetic abnormality there is significant phenotypic variability, suggesting that non-genetic elements may modify pathogenesis. Identification of such disease-modifiers is important as they might represent new therapeutic targets. A growing body of research has begun to shed light on the role played by the gut microbiome in health and disease with a number of studies linking abnormalities to ALS.

Main body: The microbiome refers to the genes belonging to the myriad different microorganisms that live within and upon us, collectively known as the microbiota. Most of these microbes are found in the intestines, where they play important roles in digestion and the generation of key metabolites including neurotransmitters. The gut microbiota is an important aspect of the environment in which our bodies operate and inter-individual differences may be key to explaining the different disease outcomes seen in ALS. Work has begun to investigate animal models of the disease, and the gut microbiomes of people living with ALS, revealing changes in the microbial communities of these groups. The current body of knowledge will be summarised in this review. Advances in microbiome sequencing methods will be highlighted, as their improved resolution now enables researchers to further explore differences at a functional level. Proposed mechanisms connecting the gut microbiome to neurodegeneration will also be considered, including direct effects via metabolites released into the host circulation and indirect effects on bioavailability of nutrients and even medications.

Conclusion: Profiling of the gut microbiome has the potential to add an environmental component to rapidly advancing studies of ALS genetics and move research a step further towards personalised medicine for this disease. Moreover, should compelling evidence of upstream neurotoxicity or neuroprotection initiated by gut microbiota emerge, modification of the microbiome will represent a potential new avenue for disease modifying therapies. For an intractable condition with few current therapeutic options, further research into the ALS microbiome is of crucial importance.
\end{abstract}

Keywords: Amyotrophic lateral sclerosis, ALS, Microbiome, Disease modifiers, Microbial metabolites, Microbial

\footnotetext{
* Correspondence: c.j.mcdermott@sheffield.ac.uk

†Sarah L. Boddy, Ilaria Giovannelli and Matilde Sassani contributed equally to this work.

'Sheffield Institute for Translational Neuroscience, University of Sheffield, Sheffield, UK

Full list of author information is available at the end of the article
}

C C The Author(s). 2021 Open Access This article is licensed under a Creative Commons Attribution 4.0 International License, which permits use, sharing, adaptation, distribution and reproduction in any medium or format, as long as you give appropriate credit to the original author(s) and the source, provide a link to the Creative Commons licence, and indicate if changes were made. The images or other third party material in this article are included in the article's Creative Commons licence, unless indicated otherwise in a credit line to the material. If material is not included in the article's Creative Commons licence and your intended use is not permitted by statutory regulation or exceeds the permitted use, you will need to obtain permission directly from the copyright holder. To view a copy of this licence, visit http://creativecommons.org/licenses/by/4.0/ The Creative Commons Public Domain Dedication waiver (http://creativecommons.org/publicdomain/zero/1.0/) applies to the data made available in this article, unless otherwise stated in a credit line to the data. 


\section{Background}

Amyotrophic lateral sclerosis (ALS) is a fatal neurodegenerative condition characterised by progressive loss of motor neurons. No effective neuroprotective therapy exists; median survival is 2 to 3 years from symptom onset, but there is considerable variation in individual outcomes [1]. Current understanding does not explain the observed heterogeneity in those affected.

In recent years, much progress has been made in elucidating genetic mutations (e.g. within C9ORF72 and SOD1) associated with ALS [2-6]. In certain cases, particular phenotypes are associated with specific genetic variants, including young age of onset [7] and cognitive impairment [8]. However, significant heterogeneity, even within families of patients suffering monogenic disease, suggests that environmental risk factors play a role. Indeed, both sporadic and monogenic ALS are thought to result from a multi-step process based on age-related incidence [9].

Some evidence for a role of specific environmental risk factors (e.g. military service) has been found [10-16]. However, most of those exposed do not develop ALS, and therefore, individual environmental factors likely contribute little to overall disease risk. Interestingly, all proposed environmental factors could potentially impact the gut microbiota and its collective functions. Therefore, the gut microbiome could represent an integrator of the overall environmental contribution to neurodegeneration development.

The most recent studies in SOD1 and C9ORF72 model mice have indicated a disease-modifying role for the gut microbiome, with disease severity correlated to particular gut microbe communities $[17,18]$. In addition to modifying the risk of developing ALS, it is also possible that a patient's microbiota can offer protective or deleterious effects, slowing or hastening disease progression, e.g. by impacting systemic inflammation.

The human microbiome refers to the collective genomes of all microbes (i.e. bacteria, archaea, viruses and fungi) that live upon or within the human body, with the community of microbes themselves known as the microbiota. A person's microbiome contains $\sim 150$-fold more genes than their human genome [19], so probing the microbiome in a meaningful way poses technical challenges. Nonetheless, recent advances in DNA sequencing technologies have allowed scientists to explore roles of resident microbial communities in health and disease.

Since most of the human microbiome resides within the intestine, several studies in common neurological conditions have focussed on the microbiome of the gastrointestinal tract. Intestinal microbial composition can impact development and progression of disease. For example, gut microbes produce biologically active metabolites and may also modify the absorption of nutrients and drug bioavailability. Particular focus has been given to a proposed bidirectional gut-brain-axis in which the gut microbiome and the CNS engage in biochemical signalling, and the role that the microbiome may play in neurodegenerative diseases such as Alzheimer's and Parkinson's diseases [20]. Most recently, investigations have begun to seek links between the gut microbiome and ALS. Small cohorts and methodology limit many of these studies. Nonetheless, differences have been identified between the gut microbiome of ALS patients and controls, supporting further research in this area.

The aim of this article is to review current literature on the microbiome and ALS, and to outline putative mechanisms by which the gut microbiome may impact on the condition. The primary research studies reviewed were selected using the following search terms: "amyotrophic lateral sclerosis" and "microbiome", "amyotrophic lateral sclerosis" and "microbial", "motor neuron disease" and "microbiome" in PubMed, up to 26 July 2020 (no lower date limit).

\section{Main text}

The gut microbiome is linked to ALS, current evidence: mouse models

The first study of an ALS mouse model identified disease-specific damage to intestinal tight junctions, increased gut permeability and reduced levels of the butyrate-producing bacteria Butyrivibrio fibrisolvens, in the SOD1 ${ }^{\text {G93A }}$ mouse [21]. Butyrate is implicated in modulating the immune response, as illustrated in the neuroinflammation section below. In 2017, the same group performed an interventional study attempting to alleviate symptoms through treatment with sodium butyrate [22]: $\mathrm{SOD} 1^{\mathrm{G} 93 \mathrm{~A}}$ mice receiving butyrate supplementation developed improved intestinal barrier function and showed delayed weight loss and death compared to untreated controls.

A recent longitudinal study of the $\mathrm{SOD} 1^{\mathrm{G} 93 \mathrm{~A}}$ model identified dysbiosis in the pre-symptomatic stage with increased within-sample variance in faecal pellets, but not in ileocolic content [23]. In contrast, an investigation into the impact of short-term vagus nerve stimulation (VNS) on the microbiome of a milder SOD1 mutant model revealed no difference between disease and WT mice at the timepoint investigated, 1 month before symptom onset [24]. No effect on the gut microbiome was seen post VNS.

A 2019 study of the gut microbiome in the SOD1 ${ }^{\text {G93A }}$ mouse model and in ALS patients represents a major step forward for the field. This is the first study to probe the functional signature of gut microbiome in ALS using shotgun metagenomics. This study identified changes in gut microbiome correlating with biological activity of a 
microbial metabolite, nicotinamide, possibly modulating the expression of mitochondrial genes in the spinal cord. Notably, detected changes correlated with disease severity in both transgenic mice and human patients [17].

Recently, a study of C9ORF72-null mice showed that loss of C9ORF72 resulted in a pro-inflammatory phenotype which was ameliorated when the gut microbial burden was reduced [18]. The GGGGCC-repeat expansion of C9ORF72 is the most common genetic cause of ALS $[5,6]$, and although this genetic change is not thought to be a pure loss-of-function mutation, reduced expression of endogenous C9ORF72 is also a feature of the human disease [25]. Nonetheless, the murine model used in this study is much less well characterised than the SOD $1^{\mathrm{G} 93 \mathrm{~A}}$ transgenic mouse model and questions have been raised on its relevance to human ALS.

Studies investigating the microbiome in mouse models of ALS are summarised in Table 1. There are key limitations to these studies, often involving small numbers of animals and 16S rRNA gene sequencing (discussed later). Caution should be drawn before extrapolating findings in mice to the human ALS due to specific limitations of the models [26] and more generally the poor translation rates of findings in rodents to humans to date [27, 28]. Regarding microbial communities, the highly controlled environments of animal facilities poorly replicate those people with ALS reside in, diverging models still further from the human situation.

\section{The gut microbiome is linked to ALS, current evidence: human studies}

Human studies of the gut microbiome in ALS have yielded equivocal findings. Two identified a reduced Firmicutes/Bacteroidetes ratio in patients $[29,30]$, whilst a third reported the opposite [31]. Nonetheless, those studies detected evidence of dysbiosis in ALS, although they utilised small cohorts $(6,5$ and 8 ALS patients, respectively) and one [30] was confined to a specific subgroup of patients with gastrointestinal symptoms. In contrast, a larger 2018 study comparing 25 patients with 32 controls discovered no substantial changes in the gut microbiome including no changes in the Firmicutes/Bacteroidetes ratio [32], though slightly higher diversity was found in ALS samples.

The largest study to date compared 50 patients and 50 age- and sex-matched controls. The abundance of various microbial genera was significantly altered in ALS compared to controls [33] and disease progression coincided with reduced microbial diversity, possibly secondary to dietary changes caused by increased disability. This highlights the importance of monitoring diet in study design. The group also trialled a 6-month probiotic intervention and found no observable benefits.
This year, one study comparing 49 probable/definite ALS cases and 50 controls found no change in the Firmicutes/Bacteroidetes ratio in ALS patients compared to controls [34]. In contrast, the most recent study found a reduced Firmicutes/Bacteroidetes ratio and increased species diversity associated with ALS samples compared to healthy controls $(n=20$, both groups) [35]. Despite finding no differences between the disease and control groups, the larger study did report that within the ALS group, a higher Firmicutes/Bacteroidetes ratio was associated with increased risk of death, as was higher species diversity [34].

Antibiotics significantly modify the balance of gut microbial species [36]; hence, correlations between antibiotic usage and seemingly unrelated diseases are of interest. A 2019 study demonstrated that the use of antibiotics, especially repeatedly, was associated with increased risk of developing ALS [37]. The study was based on whole-population data for Sweden (2006-2013 period), but limited by the retrospective design and the lack of associated microbiome data to probe for evidence of dysbiosis. Similarly, experiments using the SOD $1^{\text {G93A }}$ mouse model showed that repeated exposure to antibiotics was associated with development of a more severe motor phenotype and increased neuronal loss [17]; this effect was specific to the diseased mice and no motor effect was observed in WT mice.

All published human ALS microbiome studies are summarised in Table 2, including two studies investigating microbes residing within the CNS $[38,39]$. As those do not explore the gut microbiota, they are not discussed further in the text.

Whilst data are accumulating, many results are discordant. Studies are largely exploratory and cohort numbers have been relatively small which, in view of significant interindividual variability, may preclude identification of relevant microbiome features. These findings, alongside those reported in animal studies, demonstrate the importance of considering within-group differences across the ALS population as well as between-group changes between ALS and controls. It is possible that specific microbiome signatures may be either protective or toxic in different individuals. Future studies will need to significantly increase sample size and combine the microbiome profile with detailed measurement of interacting partners such as the host genome, nutrition and medication.

\section{A note on methodologies}

We have reviewed all published studies on the gut microbiome in ALS. Most utilised 16S rRNA gene sequencing. However, recent technological developments have made more in-depth microbiome sequencing feasible and affordable. Shotgun metagenomic sequencing is 


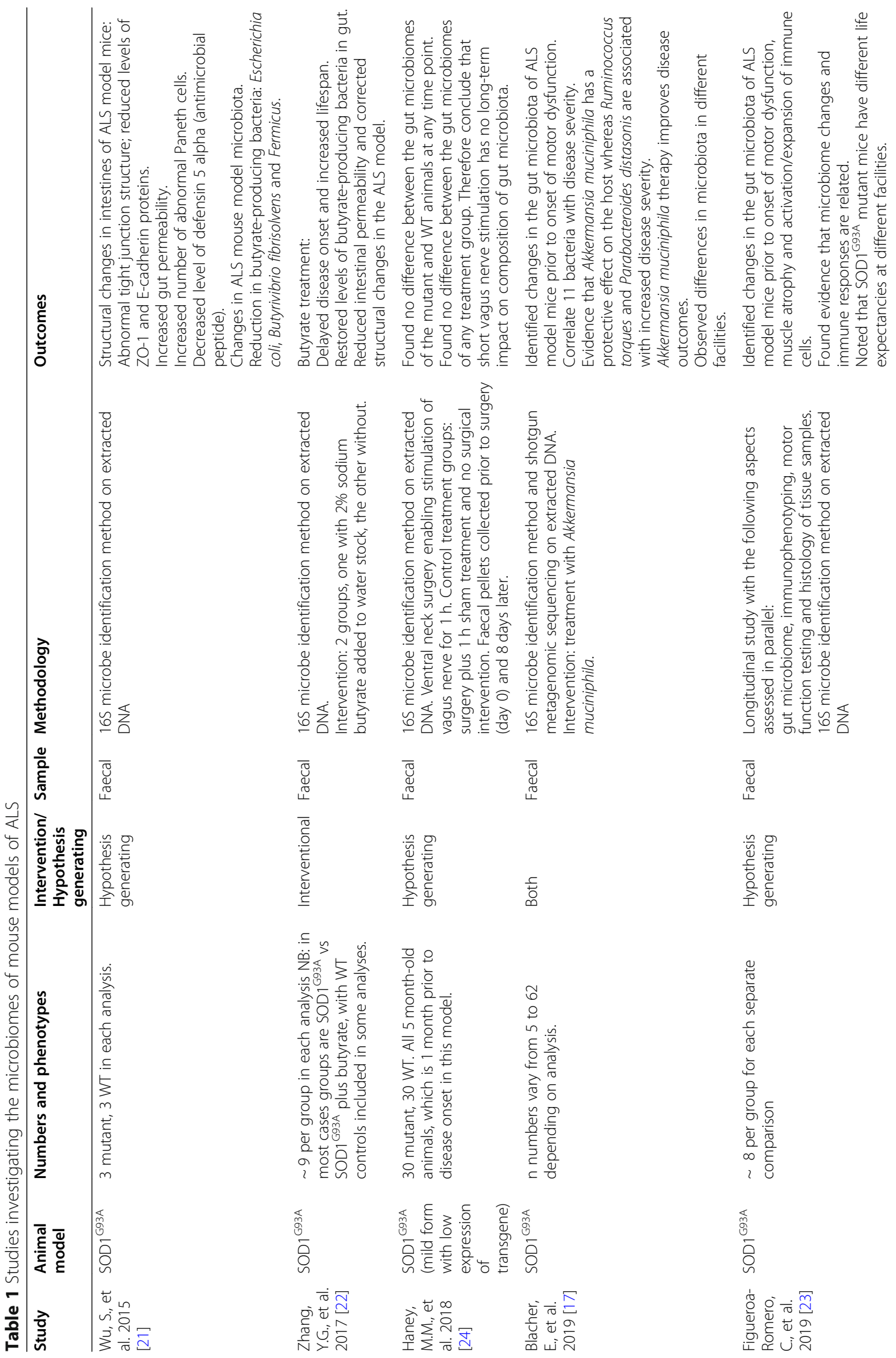




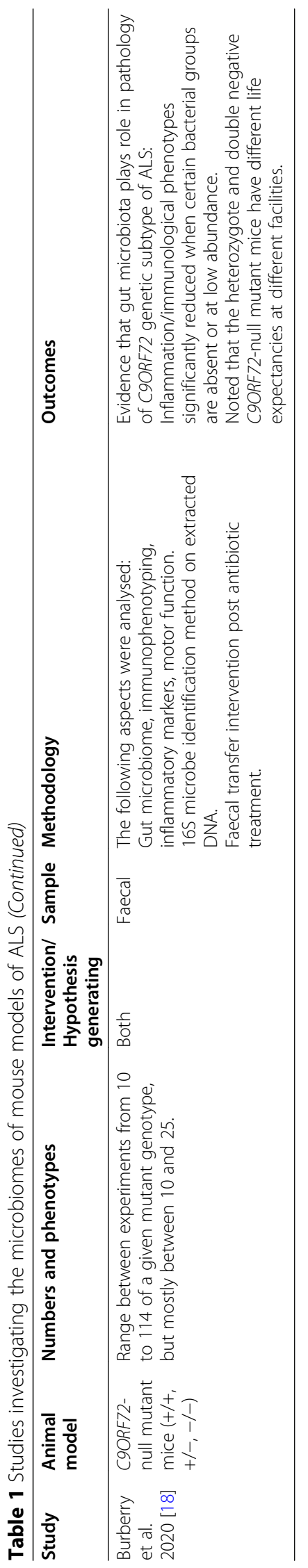




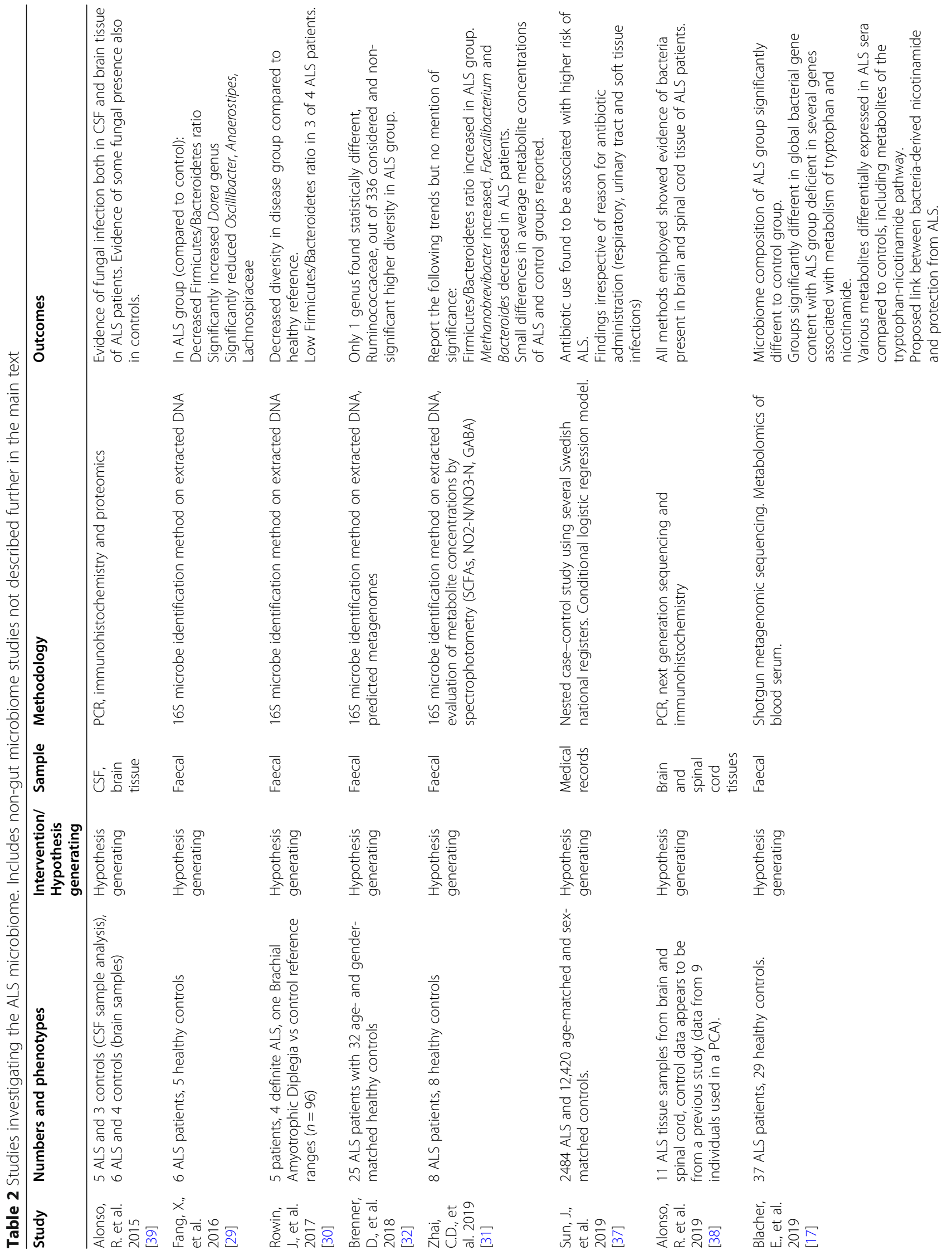




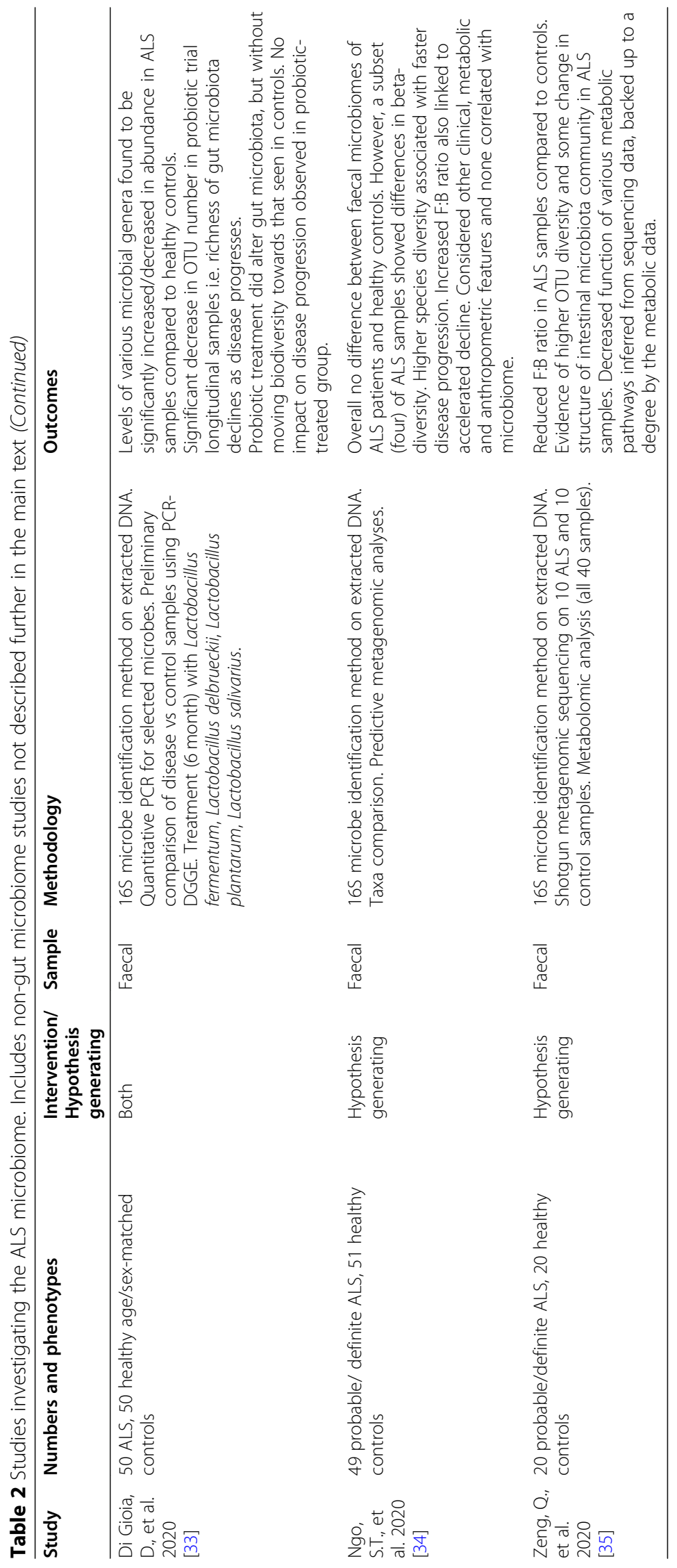


becoming the standard due to its increased resolution, enabling not only identification of microbes at species levels, but also discovery of novel microbes. Furthermore, this technology facilitates functional analyses and critically is able to account for horizontal gene-transfer events, which inferred functional analyses from 16Sderived data cannot do [40-44]. When it is considered that microbes often share functional enzymes, then a functional measure is clearly more informative than identifying specific species. We do accept that sometimes, due to cost or sample quality, 16S rRNA sequencing may be the only option and it is important to note that efforts have been made to predict function from $16 \mathrm{~S}$ data with some success [45]. The advantages offered by shotgun metagenomic sequencing are reviewed elsewhere $[44,46]$, but it is appropriate to highlight the different analytical techniques used in studies (Tables 1 and 2). Thus far, only Blacher et al. [17] have reported data based on metagenomic sequencing.

Beyond metagenomics, other methods are being developed to measure function of gut microbes including metabolomics, metatranscriptomics and metaproteomics [47]. A multi-omic approach to the microbiome is likely to improve power for detection of biology just as a multi-omic approach to the host is becoming increasingly important; this is illustrated by the use of metabolomics in the study described above [17]. Significant innovation is also occurring in analysis methods. Microbes can interact functionally even without genetransfer for example, if a collection of microbes each encodes an enzyme which produces a substrate for another enzyme carried by a different microbe. It is possible to predict the presence and effect of the so-called biosynthetic gene clusters using ClusterFinder [48].

\section{The gut microbiome may contribute to ALS heterogeneity: putative roles}

Various ways in which the gut microbiota and CNS may interact have been proposed, with communication routes referred to as the gut-brain-axis (Fig. 1). It has been suggested that microbes might influence the CNS directly, through production of neuroactive metabolites released into the systemic circulation or via the enteric nervous system [49, 50]. Possible indirect connections include modulation of CNS inflammation [51, 52], alteration of nutrient absorption [53, 54] and modification of the metabolism of exogenous drugs [55]. Evidence supporting two of these putative mechanisms has emerged from recent literature: Blacher et al. [17] provided data supporting the role of microbial neuroactive metabolites in ALS, whereas others have found links between gut microbiota, the immune system and ALS [18, 21-23]. Notably, although these mechanisms are not mutually exclusive, no consensus has yet been reached and, in addition to limitations highlighted above, studies are not directly comparable primarily because of differences in analysis methodologies. The last section will detail these two mechanisms and suggest other promising avenues yet to be explored in the field.

\section{Metabolite modulation of neuronal function}

The strongest evidence for microbe-derived neuroactive metabolites modifying ALS comes from the study of Blacher et al. [17] which focused on the protective effect of nicotinamide released by Akkermansia muciniphila (Fig. 2a). The authors argued for the role of bacterial metabolites in modulating neurodegeneration; they were able to manipulate disease severity in $\mathrm{SOD} 1^{\mathrm{G} 93 \mathrm{~A}}$ mice via supplementation with gut microbial species. Ruminococcus torques and Parabacteroides distasonis were associated with increased severity, whereas Akkermansia muciniphila improved outcomes. Mechanistic links were explored through untargeted serum metabolomicprofiling, which identified changes in biological pathways featuring nicotinamide in mice receiving Akkermansia muciniphila supplementation. Importantly, direct nicotinamide administration replicated the beneficial effects of Akkermansia muciniphila. Bulk RNA-sequencing of spinal cord tissue from SOD1 ${ }^{\text {G93A }}$ mice treated with nicotinamide or Akkermansia muciniphila revealed changes in mitochondrial function and oxidative stress pathways. These observations were corroborated in ALS patients: bacterial genes associated with tryptophan and nicotinamide synthesis were decreased in patients' stool samples, and targeted metabolomic profiling revealed a lower nicotinamide concentration in serum and CSF compared to controls.

The study authors argued for a pathway from gut microbiome function to motor neuron death via metabolites secreted into the host circulation. Nicotinamide is the precursor of NAD and NADP, coenzymes necessary for appropriate functioning of energy transducing and antioxidant pathways as well as other cellular signalling mechanisms [67], many of which have been implicated in ALS-related neurodegeneration [15, 68].

\section{Modulation of CNS inflammation}

Neuro-inflammation is a well-characterised ALS pathological mechanism, defined as a complex dysregulation amongst both resident and peripheral immunological cells [15]. Its main features are activation of microglia and astrocytes, infiltration of $\mathrm{T}$ cells and upregulation of pro-inflammatory cytokines. The human microbiome exerts a considerable influence on the immune system: in development when immune cells begin to discriminate between necessary commensals and harmful pathogens, but also in adulthood, as microbes contribute to immune homeostasis $[69,70]$. In fact, germ-free (GF) 


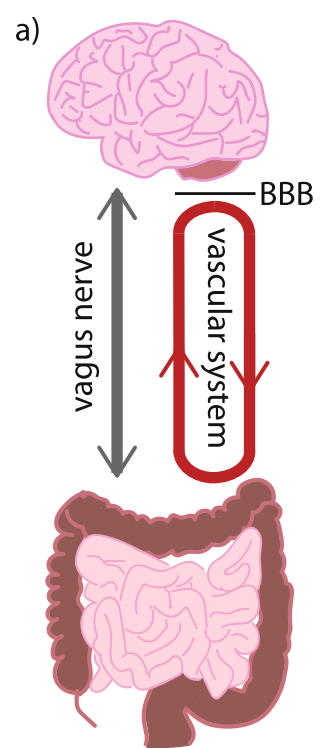

b)

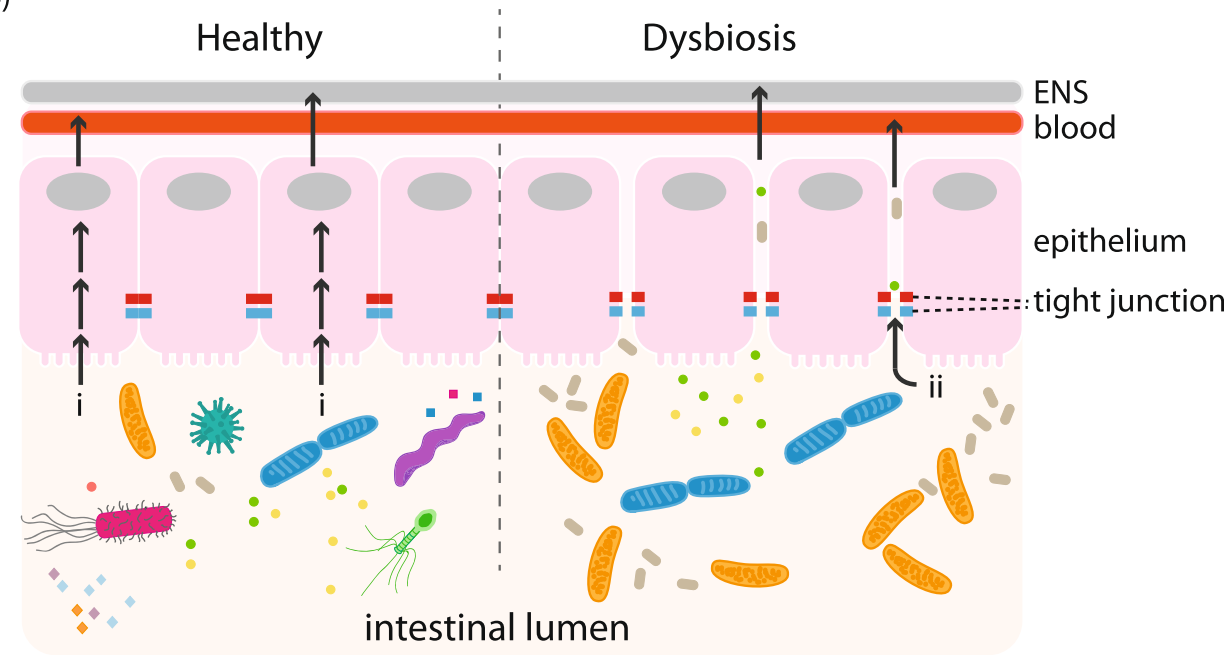

Fig. 1 Pathways linking gut microbial function to changes in the CNS. a Macro-scale pathways: The enteric nervous system (ENS) intrinsic to the gut is connected to the central nervous system directly via the vagus nerve. As such, any microbe-derived metabolite that accesses the ENS has the potential to travel to and impact the brain and spinal cord. Likewise, an extensive network of blood capillaries collects nutrients absorbed from the gut for transfer around the body. Microbial metabolites that access the bloodstream can impact any part of the body, though still need to breach the blood-brain-barrier (BBB) to access the CNS. $\mathbf{b}$ Transit across the intestinal epithelium: In a healthy gut with functional tight junctions, selective uptake of contents of the intestinal lumen occurs across the epithelial cells (route " $i$ "). Dysbiosis of the gut microbiota can damage the structural integrity of the epithelial barrier allowing uncontrolled transit of metabolites and other luminal contents to pass into the body (route "ii")

mice display a broad range of immunological abnormalities $[69,71]$. To date, the underlying mechanisms responsible for crosstalk between microbiome and the immune system are not fully understood. Several bacterial-derived molecules are thought to be immunological modulators (Fig. 2b-d) including short chain fatty acid (SCFAs), aryl hydrocarbon receptor (AHR) ligands, polyamines and polysaccharides [72]. SCFAs such as butyric acid, propionic acid and acetic acid are products of dietary fibre metabolism by the gut microbiome, mainly from Bacteroides and Firmicutes [73]. SCFAs are known to mediate regulatory $\mathrm{T}$ cell (Treg) induction through histone deacetylase inhibition (see Fig. 2). The inverse is also seen in mice where depletion of the Treg subset is associated with a significant increase in gut Firmicutes [74]. Although few studies have investigated changes in ALS microbiota compared to healthy individuals, disequilibrium of the Firmicutes/Bacteroides ratio has been reported [29-31, 33]. As these bacteria are the main producers of SCFAs, it could be speculated that alterations of these metabolites may affect ALS patients by directly acting on CNS cells and/or indirectly through immune system modulation. One of the SOD $1^{\text {G93A }}$ studies is consistent with this, where butyrate supplementation appeared to alleviate the clinical features of ALS [22]. The same group reported earlier that, prior to symptom onset, this mouse model is characterised by a leaky intestine, increased intestinal permeability and reduced abundance of butyrate-producing bacteria [21]. This is associated with increased levels of inflammatory IL-17 and abnormalities in Paneth cells, which are crucial for host-microbiome interaction and immunity [21]. Butyrate supplementation in SOD $1^{\mathrm{G} 93 \mathrm{~A}}$ mice significantly decreased intestinal permeability, reduced the number of abnormal Paneth cells and increased life span [22]. Moreover, a longitudinal study showed evidence of dysbiosis (in particular alterations in Bacteroides and Firmicutes) in SOD $1^{\mathrm{G} 93 \mathrm{~A}}$ mice beginning before disease onset (37 days) and persisting until end-stage ( 150 days). Concomitantly, alterations in the immune system were reported. These were limited to the peripheral system in early disease stages but affected the CNS later during disease course. They also documented both positive and negative correlations between microbiome dysregulation and spinal cord inflammation [23].

Microbiota-deficient mice also provide evidence linking the gut microbiome to the CNS through the immune system. Germ-free (GF) and antibiotic-treated mouse models develop immunological abnormalities and neurodegeneration [75]. In this context, microglia functions appear to be strictly connected to the gut microbiome [51] as GF or antibiotic-treated mice exhibit aberrant microglia maturation and functional impairments [76], characterised by immature phenotype, morphology alterations, diminished 


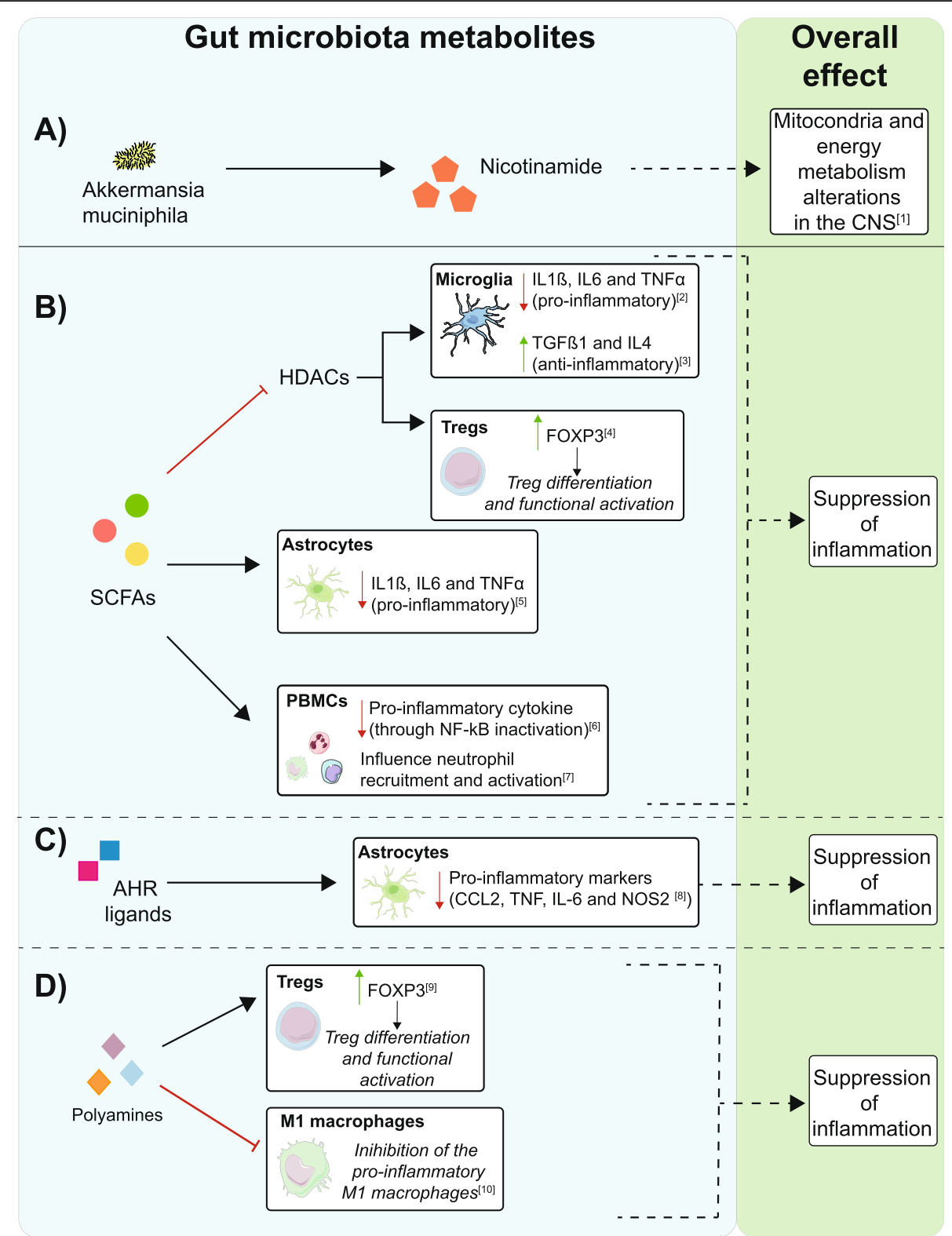

Fig. 2 Metabolites produced by microbes found within the gut can influence neuronal health either directly or indirectly via CNS inflammation. a Metabolites released by the gut microbiome can enter the system circulation where they can access the CNS; in the case of nicotinamide released by Akkermansia muciniphila, this potentially modifies energy homeostasis and oxidative stress [17]. b- $\mathbf{d}$ A number of proposed mechanisms exist by which metabolites produced by microbes found within the gut can influence the immune response and have an effect of the CNS inflammatory state. $\mathbf{b}$ Short-chain fatty acids (SCFAs) can reduce inflammation by inhibiting HDACs within microglial cells, leading to the downregulation of pro-inflammatory (IL1ß, IL6 and TNFa) and upregulation of anti-inflammatory markers (TGF $\beta$ and IL4) [56, 57]. SCFA-mediated HDAC inhibition can also impact Tregs increasing their activity via upregulation of FOXP3 [58,59]. SCFAs also influence astrocytes, reducing their inflammatory impact through downregulation of IL1B, IL6 and TNFa [60]. Lastly, SCFAs exert anti-inflammatory effects on different peripheral blood mononuclear cells: they inhibit NF-kB leading to reduced pro-inflammatory cytokine production and immune cell recruitment and activation [61-63]. c Aryl hydrocarbon receptor (AHR) ligands can modulate astrocyte activities and give rise to anti-inflammatory properties [64]. d Polyamines induce FOXP3 expression in Treg cells promoting their differentiation and activation [65]. These molecules can also inhibit inflammatory macrophages (M1) thereby preventing macrophage-induced inflammation [66]

responsiveness to lipopolysaccharides and overall attenuated immune activation. A C9ORF72-null mouse study suggests that dysregulated microflora, characterised by immunestimulating bacteria, reduce mice survival by inducing detrimental peripheral inflammation and microglia activation. However, antibiotic treatment or the transplantation of protective microbiota improved symptoms [18]. Astrocyte activity is also regulated by the gut microbiome through an 
aryl hydrocarbon receptor (AHR)-mediated mechanism involving type I interferon signalling [64]. This leads to a different hypothesis: that proinflammatory signals driven by gut microbiota may be key for physiological functioning of glia, which in turn maintain neuronal health. Abnormal function of microglia [77] and astrocytes [78] has been linked to motor neuron death. Confirmation of this hypothesis will depend on identification of direct correlations between gut microbiome features, disease severity and CNS inflammation in a single model system; it should be noted that an unbiased metabolomic study did not identify a significant inflammatory signal [17].

Given the potential link between the microbiome, the immune system and ALS, a clinical trial is currently ongoing to evaluate the effects of faecal microbial transplantation in 42 patients (NCT03766321). The investigators anticipate that microbiome modulation will increase the proportion and suppressive abilities of immunesuppressive Treg cells leading to the establishment of a neuroprotective anti-inflammatory environment [79]. Moreover, several experimental medicine trials are ongoing with the aim of modulating the immune response using small molecules (e.g. MIROCALS (NCT03039673)) [80]. Should analogous results be achieved by altering the composition of the gut microbiome, this would be an attractive alternative approach.

\section{Impact on nutrition}

Much research has focused on the role of the gut microbiome in human nutrition [81]. Microbes resident in the gut can alter the quantity of nutrients extracted from food and even synthesise key nutrients themselves, including vitamin $K$ and various $B$ vitamins [82]. Experiments transplanting the gut microbiota of obese mice or humans into germ-free animals of a healthy weight produced weight-gain demonstrating that metabolic phenotypes can be transmitted via the gut microbiome [83, 84]. Weight maintenance has been highlighted as critical to the clinical outcome in ALS, with rapid weight loss associated with faster disease progression [85]. Ongoing work is focused on modifying the diet in patients with a view to slowing disease progression. It is possible that, ultimately, this work will need to investigate both diet and the modulating effect of the gut microbiome on nutrition. Furthermore, Di Gioia et al. showed a decline in microbial diversity in ALS samples over the course of a probiotic study, the only longitudinal microbiome data reported in this disease group to date [33]. This is not surprising as the progression of ALS is associated with declining chewing, swallowing and self-feeding functions, all of which are likely to impact the gut microbiota, in addition to reduced physical activity and likely environmental changes e.g. associated with leaving the workplace and/or becoming increasingly house-bound.
Indeed, during the later stages of the disease, many patients opt to be fed enterally through formulated foods, a dramatic dietary change likely to impact the microbial communities of the gut. Therefore, it is also important to consider the impact disease progression has on the gut microbiota. This is critical when considering differences seen between ALS and control samples, with caution necessary when inferring aspects of the microbiota may play a role in disease development.

\section{Impact on drug efficacy}

The gut microbiome can also impact disease via metabolism of enterally delivered drugs. A recent study revealed that the primary treatment for Parkinson's disease, L-DOPA, is metabolised by gut microbial species which vary in abundance between individuals [55]. Prevalence of a microbial gene encoding an enzyme proficient in decarboxylating L-DOPA correlated positively with the drug dosage required to provide symptomatic relief and with L-DOPA concentration in the systemic circulation. In 2019, a study assessed the capacity of a panel of gut bacteria to metabolise a selection of commonly prescribed drugs including Riluzole, the only drug that has shown to confer a survival benefit in ALS [86]. Riluzole was significantly metabolised by 40 of the bacteria screened [87] many of which are known to vary in prevalence in the human population. The plasma concentration of Riluzole reportedly shows low withinpatient variability compared to relatively high interpatient variability [88], which is not explained by differences in metabolism after gut absorption [89]. Modification of Riluzole bioavailability by the gut microbiome may explain the observed interpatient variability in plasma levels.

\section{Impact on non-motor ALS symptoms}

Finally, the microbiome has been linked to other symptoms known to impact subgroups of ALS patients, such as depression, anxiety and constipation [90]. The gut microbiota can produce various peptides and neurotransmitters that could directly impact mood $[49,90]$ whilst the brain affects the gut through a variety of mechanisms including stress responses [91]. Unravelling the role the gut microbiota play in regulating brain function relating to neuropsychiatric conditions has only just begun [90, 92], but there is potential for this to be a means of improving quality of life for ALS patients.

Regarding constipation, another symptom often reported by ALS patients [93], roles for the microbiome in luminal fluid (metabolism of bile acids [94], generation of short chain fatty acids $[95,96]$ and methane production [97]) as well as mucosal layer of the colon [98] in regulating the absorption of fluids into the bloodstream have all been proposed. Improved management of these 
symptoms would improve quality of life [99] irrespective of disease progression.

\section{Integration of microbiome with host genomics}

Notably, the gut microbiome is affected by environment, and evidence suggests almost complete independence from host genetics [100]; therefore, the two measures may be usefully combined to describe gene-environment interaction. This could be a powerful tool to approach diseases resulting from a complex interaction of genes and environment. Approaches such as deep learning or other machine learning methodologies may be required to overcome non-linearity. There is potential to provide a personalised medicine approach whereby interventions targeted at the microbiome are tailored to the host's genome.

\section{Conclusion}

\section{The missing piece of the puzzle?}

It is well established that people with ALS exhibit a wide range of disease severity and whilst some risk factors have been identified, they remain insufficient to fully explain this heterogeneity. The gut microbiome may be crucial in resolving some of these differences due to the variety of ways it could affect disease, both directly and indirectly. Further research is essential to identify relevant microbial players in ALS so that they may be targeted in future therapies seeking to modify gut microbiota to modulate disease progression and improve quality of life. It is likely that such interventions will be personally tailored to patients from different environments and with different genotypes.

\section{Acknowledgements}

None.

\section{Authors' contributions}

S L Boddy, I Giovannelli and M Sassani all contributed equally to producing this manuscript and should share the first authorship. C J McDermott should be considered the corresponding author. C J McDermott conceived the idea and general approach for the review. S L Boddy, I Giovannelli, M Sassani and J Cooper-Knock conducted the literature search and produced the first draft. C J McDermott, P J Shaw, L A Barker, E Elinav, E Segal and M P Snyder critically reviewed and edited subsequent drafts. All authors read and approved the final manuscript.

\section{Funding}

All funders are listed in the "Funding Information" section of the online submission system.

\section{Availability of data and materials}

Not applicable (Review).

\section{Ethics approval and consent to participate}

As this article is a review of other studies, ethical approval and consent to participate do not apply to this document.

\section{Consent for publication}

All authors give consent for the publication of the manuscript.

\section{Competing interests}

The authors have no competing interests.

\section{Author details}

${ }^{1}$ Sheffield Institute for Translational Neuroscience, University of Sheffield, Sheffield, UK. ${ }^{2}$ Stanford Center for Genomics and Personalized Medicine, Stanford University School of Medicine, Stanford, USA. ${ }^{3}$ Department of Computer Science and Applied Mathematics, Weizmann Institute of Science, Rehovot, Israel. ${ }^{4}$ Department of Immunology, Weizmann Institute of Science, Rehovot, Israel. ${ }^{5}$ Division of Cancer-Microbiome Research, DKFZ, Heidelberg, Germany. ${ }^{6}$ Centre for Behavioural Science and Applied Psychology, Sheffield Hallam University, Sheffield, UK.

Received: 13 August 2020 Accepted: 9 December 2020

Published online: 20 January 2021

\section{References}

1. Chio A, Logroscino G, Hardiman O, Swingler R, Mitchell D, Beghi E, et al. Prognostic factors in ALS: a critical review. Amyotroph Lateral Scler. 2009;10: 310-23.

2. Rosen DR, Siddique T, Patterson D, Figlewicz DA, Sapp P, Hentati A, et al. Mutations in $\mathrm{Cu} / \mathrm{Zn}$ superoxide dismutase gene are associated with familial amyotrophic lateral sclerosis. Nature. 1993;362:59-62.

3. Kwiatkowski TJ Jr, Bosco DA, Leclerc AL, Tamrazian E, Vanderburg CR, Russ C, et al. Mutations in the FUS/TLS gene on chromosome 16 cause familial amyotrophic lateral sclerosis. Science. 2009;323:1205-8.

4. Sreedharan J, Blair IP, Tripathi VB, Hu X, Vance C, Rogelj B, et al. TDP-43 mutations in familial and sporadic amyotrophic lateral sclerosis. Science. 2008:319:1668-72

5. DeJesus-Hernandez M, Mackenzie IR, Boeve BF, Boxer AL, Baker M, Rutherford NJ, et al. Expanded GGGGCC hexanucleotide repeat in noncoding region of C9ORF72 causes chromosome 9p-linked FTD and ALS. Neuron. 2011;72:245-56

6. Renton AE, Majounie E, Waite A, Simón-Sánchez J, Rollinson S, Gibbs JR, et al. A hexanucleotide repeat expansion in C9ORF72 is the cause of chromosome 9p21-linked ALS-FTD. Neuron. 2011;72:257-68.

7. Zou Z-Y, Liu M-S, Li X-G, Cui L-Y. Mutations in SOD1 and FUS caused juvenile-onset sporadic amyotrophic lateral sclerosis with aggressive progression. Ann Transl Med. 2015;3 https://www.ncbi.n/m.nih.gov/pmc/ articles/pmc4583590/.

8. Cooper-Knock J, Shaw PJ, Kirby J. The widening spectrum of C9ORF72related disease; genotype/phenotype correlations and potential modifiers of clinical phenotype. Acta Neuropathol. 2014;127:333-45.

9. Chiò A, Mazzini L, D'Alfonso S, Corrado L, Canosa A, Moglia C, et al. The multistep hypothesis of ALS revisited. Neurology. 2018;91:e635-42. https:// doi.org/10.1212/wnl.0000000000005996.

10. Andrew AS, Caller TA, Tandan R, Duell EJ, Henegan PL, Field NC, et al. Environmental and occupational exposures and amyotrophic lateral sclerosis in New England. Neurodegener Dis. 2017;17:110-6.

11. Banack SA, Cox PA. Biomagnification of cycad neurotoxins in flying foxes. Neurology. 2003;61:387-9. https://doi.org/10.1212/01.wnl.0000078320.18564. gf.

12. Banack SA, Murch SJ, Cox PA. Neurotoxic flying foxes as dietary items for the Chamorro people, Marianas Islands. J Ethnopharmacol. 2006;106:97-104. https://doi.org/10.1016/j.jep.2005.12.032.

13. Beard JD, Kamel F. Military service, deployments, and exposures in relation to amyotrophic lateral sclerosis etiology and survival. Epidemiol Rev. 2015; 37:55-70.

14. Fang F, Quinlan P, Ye W, Barber MK, Umbach DM, Sandler DP, et al. Workplace exposures and the risk of amyotrophic lateral sclerosis. Environ Health Perspect. 2009;117:1387-92.

15. Cooper-Knock J, Jenkins T, Shaw PJ. Clinical and molecular aspects of motor neuron disease. Morgan \& Claypool Life Sciences: Biota Publishing; 2013. https://doi.org/10.4199/C00093ED1V01Y201309GMM004.

16. Su F-C, Goutman SA, Chernyak S, Mukherjee B, Callaghan BC, Batterman S, et al. Association of environmental toxins with amyotrophic lateral sclerosis. JAMA Neurol. 2016;73:803-11.

17. Blacher E, Bashiardes S, Shapiro H, Rothschild D, Mor U, Dori-Bachash M, et al. Potential roles of gut microbiome and metabolites in modulating ALS in mice. Nature. 2019;572:474-80. 
18. Burberry A, Wells MF, Limone F, Couto A, Smith KS, Keaney J, et al. C9orf72 suppresses systemic and neural inflammation induced by gut bacteria. Nature. 2020;582:89-94.

19. Qin J, Li R, Raes J, Arumugam M, Burgdorf KS, Manichanh C, et al. A human gut microbial gene catalogue established by metagenomic sequencing. Nature. 2010;464:59-65.

20. Bell JS, Spencer Jl, Yates RL, Yee SA, Jacobs BM, DeLuca GC. Invited Review: From nose to gut - the role of the microbiome in neurological disease. Neuropathol Appl Neurobiol. 2019;45:195-215. https://doi.org/10.1111/nan 12520.

21. Wu S, Yi J, Zhang Y-G, Zhou J, Sun J. Leaky intestine and impaired microbiome in an amyotrophic lateral sclerosis mouse model. Physiol Rep. 2015;3:e12356. https://doi.org/10.14814/phy2.12356.

22. Zhang Y-G, Wu S, Yi J, Xia Y, Jin D, Zhou J, et al. Target intestinal microbiota to alleviate disease progression in amyotrophic lateral sclerosis. Clin Ther. 2017;39:322-36. https://doi.org/10.1016/j.clinthera.2016.12.014.

23. Figueroa-Romero C, Guo K, Murdock BJ, Paez-Colasante X, Bassis CM, Mikhail KA, et al. Temporal evolution of the microbiome, immune system and epigenome with disease progression in ALS mice. Dis Models Mech. 2020;13:dmm041947. https://doi.org/10.1242/dmm.041947.

24. Haney MM, Ericsson AC, Lever TE. Effects of intraoperative vagal nerve stimulation on the gastrointestinal microbiome in a mouse model of amyotrophic lateral sclerosis. Comp Med. 2018;68:452-60.

25. Dickson DW, Baker MC, Jackson JL, DeJesus-Hernandez M, Finch NA, Tian S, et al. Extensive transcriptomic study emphasizes importance of vesicular transport in C9orf72 expansion carriers. Acta Neuropathol Commun. 2019;7:150.

26. Benatar M. Lost in translation: treatment trials in the SOD1 mouse and in human ALS. Neurobiol Dis. 2007;26:1-13.

27. Waring MJ, Arrowsmith J, Leach AR, Leeson PD, Mandrell S, Owen RM, et al. An analysis of the attrition of drug candidates from four major pharmaceutical companies. Nat Rev Drug Discov. 2015;14:475-86.

28. Mak IW, Evaniew N, Ghert M. Lost in translation: animal models and clinical trials in cancer treatment. Am J Transl Res. 2014;6:114-8.

29. Fang X, Wang X, Yang S, Meng F, Wang X, Wei H, et al. Evaluation of the microbial diversity in amyotrophic lateral sclerosis using high-throughput sequencing. Front Microbiol. 2016;7. https://doi.org/10.3389/fmicb.2016. 01479.

30. Rowin J, Xia Y, Jung B, Sun J. Gut inflammation and dysbiosis in human motor neuron disease. Physiol Rep. 2017;5:e13443. https://doi.org/10.14814/ phy2.13443.

31. Zhai C-D, Zheng J-J, An B-C, Huang H-F, Tan Z-C. Intestinal microbiota composition in patients with amyotrophic lateral sclerosis. Chin Med J. 2019;132:1815-22. https://doi.org/10.1097/cm9.0000000000000351.

32. Brenner D, Hiergeist A, Adis C, Mayer B, Gessner A, Ludolph AC, et al. The fecal microbiome of ALS patients. Neurobiol Aging. 2018;61:132-7. https:// doi.org/10.1016/j.neurobiolaging.2017.09.023.

33. Di Gioia D, Cionci NB, Baffoni L, Amoruso A, Pane M, et al. A prospective longitudinal study on the microbiota composition in amyotrophic lateral sclerosis. BMC Med. 2020;18. https://doi.org/10.1186/ s12916-020-01607-9.

34. Ngo ST, Restuadi R, McCrae AF, Van Eijk RP, Garton F, Henderson RD, Wray NR, McCombe PA, Steyn FJ. Progression and survival of patients with motor neuron disease relative to their fecal microbiota. Amyotrophic Lateral Sclerosis and Frontotemporal Degeneration. 2020;21:7-8:549-62. https://doi.org/10. 1080/21678421.2020.1772825.

35. Zeng Q, Shen J, Chen K, Zhou J, Liao Q, Lu K, et al. The alteration of gut microbiome and metabolism in amyotrophic lateral sclerosis patients. Sci Rep. 2020;10:12998.

36. Willing BP, Russell SL, Finlay BB. Shifting the balance: antibiotic effects on host-microbiota mutualism. Nat Rev Microbiol. 2011;9:233-43.

37. Sun J, Zhan Y, Mariosa D, Larsson H, Almqvist C, Ingre C, et al. Antibiotics use and risk of amyotrophic lateral sclerosis in Sweden. Eur J Neurol. 2019; 26:1355-61. https://doi.org/10.1111/ene.13986.

38. Alonso R, Pisa D, Marina Al, Morato E, Rábano A, Rodal I, et al. Evidence for fungal infection in cerebrospinal fluid and brain tissue from patients with amyotrophic lateral sclerosis. Int J Biol Sci. 2015;11:546-58. https://doi.org/ 10.7150/ijbs.11084

39. Alonso R, Pisa D, Carrasco L. Searching for bacteria in neural tissue from amyotrophic lateral sclerosis. Front Neurosci. 2019;13. https://doi.org/10. 3389/fnins.2019.00171.
40. Lerner A, Matthias T, Aminov R. Potential effects of horizontal gene exchange in the human gut. Front Immunol. 2017;8:1630.

41. Liu L, Chen X, Skogerbø G, Zhang P, Chen R, He S, et al. The human microbiome: a hot spot of microbial horizontal gene transfer. Genomics. 2012;100:265-70.

42. Sitaraman R. Prokaryotic horizontal gene transfer within the human holobiont: ecological-evolutionary inferences, implications and possibilities. Microbiome. 2018;6:163.

43. Jeong H, Arif B, Caetano-Anollés G, Kim KM, Nasir A. Horizontal gene transfer in human-associated microorganisms inferred by phylogenetic reconstruction and reconciliation. Sci Rep. 2019;9:5953.

44. Jovel J, Patterson J, Wang W, Hotte N, O'Keefe S, Mitchel T, et al. Characterization of the gut microbiome using $16 \mathrm{~S}$ or shotgun metagenomics. Front Microbiol. 2016;7:459.

45. Langille MGl, Zaneveld J, Caporaso JG, McDonald D, Knights D, Reyes $J A$, et al. Predictive functional profiling of microbial communities using 16S rRNA marker gene sequences. Nat Biotechnol. 2013;31:81421.

46. Mas-Lloret J, Obón-Santacana M, Ibáñez-Sanz G, Guinó E, Pato ML, Rodriguez-Moranta F, et al. Gut microbiome diversity detected by highcoverage $16 \mathrm{~S}$ and shotgun sequencing of paired stool and colon sample. Sci Data. 2020;7:92.

47. Tilocca B, Pieroni L, Soggiu A, Britti D, Bonizzi L, Roncada P, et al. Gut-brain axis and neurodegeneration: state-of-the-art of meta-omics sciences for microbiota characterization. Int J Mol Sci. 2020;21:4045.

48. Cimermancic P, Medema MH, Claesen J, Kurita K, Wieland Brown LC, Mavrommatis $\mathrm{K}$, et al. Insights into secondary metabolism from a global analysis of prokaryotic biosynthetic gene clusters. Cell. 2014; 158:412-21.

49. Mittal R, Debs LH, Patel AP, Nguyen D, Patel K, O'Connor G, et al. Neurotransmitters: the critical modulators regulating gut-brain axis. J Cell Physiol. 2017;232:2359-72.

50. De Vadder F, Kovatcheva-Datchary P, Goncalves D, Vinera J, Zitoun C, Duchampt A, et al. Microbiota-generated metabolites promote metabolic benefits via gut-brain neural circuits. Cell. 2014;156:84-96.

51. Fung TC, Olson CA, Hsiao EY. Interactions between the microbiota, immune and nervous systems in health and disease. Nat Neurosci. 2017;20:145-55. https://doi.org/10.1038/nn.4476.

52. Ma Q, Xing C, Long W, Wang HY, Liu Q, Wang R-F. Impact of microbiota on central nervous system and neurological diseases: the gut-brain axis. J Neuroinflammation. 2019;16:53.

53. Rowland I, Gibson G, Heinken A, Scott K, Swann J, Thiele I, et al. Gut microbiota functions: metabolism of nutrients and other food components. Eur J Nutr. 2018;57:1-24.

54. Krajmalnik-Brown R, llhan Z-E, Kang D-W, DiBaise JK. Effects of gut microbes on nutrient absorption and energy regulation. Nutr Clin Pract. 2012;27:201-14.

55. van Kessel SP, Frye AK, El-Gendy AO, Castejon M, Keshavarzian A, van Dijk G, et al. Gut bacterial tyrosine decarboxylases restrict levels of levodopa in the treatment of Parkinson's disease. Nat Commun. 2019;10. https://doi.org/10. 1038/s41467-019-08294-y.

56. Wang P, Zhang Y, Gong Y, Yang R, Chen Z, Hu W, et al. Sodium butyrate triggers a functional elongation of microglial process via Akt-small RhoGTPase activation and HDACs inhibition. Neurobiol Dis. 2018;111:12-25.

57. Soliman ML, Puig KL, Combs CK, Rosenberger TA. Acetate reduces microglia inflammatory signaling in vitro. J Neurochem. 2012;123:555-67.

58. Furusawa Y, Obata Y, Fukuda S, Endo TA, Nakato G, Takahashi D, et al. Commensal microbe-derived butyrate induces the differentiation of colonic regulatory T cells. Nature. 2013;504:446-50.

59. Arpaia N, Campbell C, Fan X, Dikiy S, van der Veeken J, deRoos P, et al. Metabolites produced by commensal bacteria promote peripheral regulatory T-cell generation. Nature. 2013;504:451-5.

60. Soliman ML, Combs CK, Rosenberger TA. Modulation of inflammatory cytokines and mitogen-activated protein kinases by acetate in primary astrocytes. J Neurolmmune Pharmacol. 2013;8:287-300.

61. Zhang D, Frenette PS. Cross talk between neutrophils and the microbiota. Blood. 2019;133:2168-77.

62. Usami M, Kishimoto K, Ohata A, Miyoshi M, Aoyama M, Fueda Y, et al. Butyrate and trichostatin $A$ attenuate nuclear factor $\mathrm{kB}$ activation and tumor necrosis factor a secretion and increase prostaglandin E2 secretion in human peripheral blood mononuclear cells. Nutr Res. 2008;28:321-8. https://doi.org/10.1016/j.nutres.2008.02.012. 
63. Vinolo MAR, Rodrigues HG, Hatanaka E, Sato FT, Sampaio SC, Curi R. Suppressive effect of short-chain fatty acids on production of proinflammatory mediators by neutrophils. J Nutr Biochem. 2011;22:849-55.

64. Rothhammer V, Mascanfroni ID, Bunse L, Takenaka MC, Kenison JE, Mayo L, et al. Type I interferons and microbial metabolites of tryptophan modulate astrocyte activity and central nervous system inflammation via the aryl hydrocarbon receptor. Nat Med. 2016;22:586-97.

65. Carriche GM, Almeida L, Stüve P, Velasquez L, Dhillon-LaBrooy A, Roy U, et al. Regulating T-cell differentiation through the polyamine spermidine. J Allergy Clin Immunol. 2020. https://doi.org/10.1016/j.jaci.2020.04.037.

66. Zhang M, Wang H, Tracey KJ. Regulation of macrophage activation and inflammation by spermine: a new chapter in an old story. Crit Care Med. 2000;28(4 Suppl):N60-6.

67. Gasperi V, Sibilano M, Savini I, Catani MV. Niacin in the central nervous system: an update of biological aspects and clinical applications. Int J Mol Sci. 2019;20. https://doi.org/10.3390/ijms20040974.

68. Smith EF, Shaw PJ, De Vos KJ. The role of mitochondria in amyotrophic lateral sclerosis. Neurosci Lett. 2019;710:132933.

69. Gensollen T, lyer SS, Kasper DL, Blumberg RS. How colonization by microbiota in early life shapes the immune system. Science. 2016:352:53944. https://doi.org/10.1126/science.aad9378.

70. Belkaid $Y$, Hand TW. Role of the microbiota in immunity and inflammation. Cell. 2014;157:121-41. https://doi.org/10.1016/j.cell.2014.03.011.

71. Vangoitsenhoven R, Cresci GAM. Role of microbiome and antibiotics in autoimmune diseases. Nutr Clin Pract. 2020;35:406-16. https://doi.org/10. 1002/ncp.10489

72. Rooks MG, Garrett WS. Gut microbiota, metabolites and host immunity. Nat Rev Immunol. 2016;16:341-52. https://doi.org/10.1038/nri.2016.42.

73. Feng $\mathrm{W}, \mathrm{Ao} \mathrm{H}$, Peng $\mathrm{C}$. Gut microbiota, short-chain fatty acids, and herbal medicines. Front Pharmacol. 2018;9. https://doi.org/10.3389/fphar.2018. 01354.

74. Kehrmann J, Effenberg L, Wilk C, Schoemer D, Phuong NNT, Adamczyk A, et al. Depletion of Foxp3 regulatory $T$ cells is accompanied by an increase in the relative abundance of Firmicutes in the murine gut microbiome. Immunology. 2020;159:344-53. https://doi.org/10.1111/imm.13158.

75. Sampson TR, Mazmanian SK. Control of brain development, function, and behavior by the microbiome. Cell Host Microbe. 2015;17:565-76. https://doi. org/10.1016/j.chom.2015.04.011

76. Erny D, Hrabě de Angelis AL, Jaitin D, Wieghofer P, Staszewski O, David E, et al. Host microbiota constantly control maturation and function of microglia in the CNS. Nat Neurosci. 2015;18:965-77.

77. Cooper-Knock J, Green C, Altschuler G, Wei W, Bury JJ, Heath PR, et al. A data-driven approach links microglia to pathology and prognosis in amyotrophic lateral sclerosis. Acta Neuropathol Commun. 2017;5:23.

78. Meyer K, Ferraiuolo L, Miranda CJ, Likhite S, McElroy S, Renusch S, et al. Direct conversion of patient fibroblasts demonstrates non-cell autonomous toxicity of astrocytes to motor neurons in familial and sporadic ALS. Proc Natl Acad Sci U S A. 2014;111:829-32.

79. Mandrioli J, Amedei A, Cammarota G, Niccolai E, Zucchi E, D'Amico R, et al. FETR-ALS study protocol: a randomized clinical trial of fecal microbiota transplantation in amyotrophic lateral sclerosis. Front Neurol. 2019;10:1021

80. MIROCALS: Modifying Immune Response and OutComes in ALS - Full Text View - ClinicalTrials.gov. https://clinicaltrials.gov/ct2/show/NCT03039673. Accessed 19 July 2020.

81. Zmora N, Suez J, Elinav E. You are what you eat: diet, health and the gut microbiota. Nat Rev Gastroenterol Hepatol. 2019;16:35-56.

82. Mills M, Stanton L, Smith R. Precision Nutrition and the Microbiome, Part I: Current State of the Science. Nutrients. 2019;11:923. https://doi.org/10.3390/ nu11040923.

83. Ridaura VK, Faith JJ, Rey FE, Cheng J, Duncan AE, Kau AL, et al. Gut microbiota from twins discordant for obesity modulate metabolism in mice. Science. 2013;341:1241214

84. Turnbaugh PJ, Ley RE, Mahowald MA, Magrini V, Mardis ER, Gordon JI. An obesity-associated gut microbiome with increased capacity for energy harvest. Nature. 2006;444:1027-31. https://doi.org/10.1038/nature05414.

85. Stambler N, Charatan M, Cedarbaum JM, ALS CNTF Treatment Study Group*. Prognostic indicators of survival in ALS. Neurology. 1998;50: $66-72$.
86. Bensimon $G$, Lacomblez $L$, Meininger $V$. A controlled trial of riluzole in amyotrophic lateral sclerosis. ALS/Riluzole Study Group. N Engl J Med. 1994; 330:585-91.

87. Zimmermann M, Zimmermann-Kogadeeva M, Wegmann R, Goodman AL. Mapping human microbiome drug metabolism by gut bacteria and their genes. Nature. 2019;570:462-7. https://doi.org/10.1038/ s41586-019-1291-3.

88. Groeneveld GJ, van Kan HJM, Sastre Toraño J, Veldink JH, Guchelaar HJ, Wokke JHJ, et al. Inter- and intraindividual variability of riluzole serum concentrations in patients with ALS. J Neurol Sci. 2001;191:121-5. https://doi.org/10.1016/s0022-510x(01)00613-x.

89. Zarate CA, Manji HK. Riluzole in psychiatry: a systematic review of the literature. Expert Opin Drug Metab Toxicol. 2008:4:1223-34. https://doi.org/ 10.1517/17425255.4.9.1223.

90. Lach G, Schellekens H, Dinan TG, Cryan JF. Anxiety, depression, and the microbiome: a role for gut peptides. Neurotherapeutics. 2018;15: 36-59.

91. Karl JP, Hatch AM, Arcidiacono SM, Pearce SC, Pantoja-Feliciano IG, Doherty $L A$, et al. Effects of psychological, environmental and physical stressors on the gut microbiota. Front Microbiol. 2018;9:2013.

92. Cheung SG, Goldenthal AR, Uhlemann A-C, Mann JJ, Miller JM, Sublette ME. Systematic review of gut microbiota and major depression. Front Psychiatry. 2019;10:34.

93. Jackson CE, McVey AL, Rudnicki S, Dimachkie MM, Barohn RJ. Symptom management and end-of-life care in amyotrophic lateral sclerosis. Neurol Clin. 2015;33:889-908.

94. Abrahamsson H, Ostlund-Lindqvist A-M, Nilsson R, Simrén M, Gillberg P-G. Altered bile acid metabolism in patients with constipation-predominant irritable bowel syndrome and functional constipation. Scand J Gastroenterol. 2008:43:1483-8.

95. Yajima T. Contractile effect of short-chain fatty acids on the isolated colon of the rat. J Physiol. 1985;368:667-78.

96. Zhuang M, Shang W, Ma Q, Strappe P, Zhou Z. Abundance of probiotics and butyrate-production microbiome manages constipation via short-chain fatty acids production and hormones secretion. Mol Nutr Food Res. 2019;63: 1801187. https://doi.org/10.1002/mnfr.201801187.

97. Pimentel $M$, Lin $H C$, Enayati $P$, van den Burg $B$, Lee $H-R$, Chen JH, et al. Methane, a gas produced by enteric bacteria, slows intestinal transit and augments small intestinal contractile activity. Am J Physiol Gastrointest Liver Physiol. 2006;290:G1089-95. https://doi.org/10.1152/ajpgi.00574.2004.

98. Parthasarathy G, Chen J, Chen X, Chia N, O'Connor HM, Wolf PG, et al. Relationship between microbiota of the colonic mucosa vs feces and symptoms, colonic transit, and methane production in female patients with chronic constipation. Gastroenterology. 2016;150:367-79.e1.

99. Hobson EV, McDermott CJ. Supportive and symptomatic management of amyotrophic lateral sclerosis. Nat Rev Neurol. 2016;12:526-38.

100. Rothschild D, Weissbrod O, Barkan E, Kurilshikov A, Korem T, Zeevi D, et al. Environment dominates over host genetics in shaping human gut microbiota. Nature. 2018;555:210-5.

\section{Publisher's Note}

Springer Nature remains neutral with regard to jurisdictional claims in published maps and institutional affiliations.

\section{Ready to submit your research? Choose BMC and benefit from:}

- fast, convenient online submission

- thorough peer review by experienced researchers in your field

- rapid publication on acceptance

- support for research data, including large and complex data types

- gold Open Access which fosters wider collaboration and increased citations

- maximum visibility for your research: over $100 \mathrm{M}$ website views per year

At BMC, research is always in progress.

Learn more biomedcentral.com/submissions 